EPJ Web of Conferences 66, 07020 (2014)

DOI: $10.1051 /$ epjconf/ 20146607020

(C) Owned by the authors, published by EDP Sciences, 2014

\title{
Protoquark stars: stability windows and magnetic field effects
}

\author{
Débora Peres Menezes ${ }^{1, a}$, Verônica Dexheimer ${ }^{2}$, and James Rudinei Torres ${ }^{1}$ \\ ${ }^{1}$ Depto de Física - CFM - Universidade Federal de Santa Catarina Florianópolis - SC - CP. 476 - CEP 88.040 \\ - 900 - Brazil \\ ${ }^{2}$ Department of Physics, Kent State University, Kent $\mathrm{OH} 44242$, USA
}

\begin{abstract}
Recently the measurement of (at least) two very massive neutron stars plus the theoretical predictions related to the effects of magnetic fields on the QCD phase diagram shed a new light on the possibility that magnetars can be constituted of deconfined quark matter only. In this work we revisit the conditions for stable quark matter by investigating stability windows for different models at zero and finite temperature and checking the resulting stellar macroscopic properties. Once magnetic fields are considered, investigating anisotropy in the pressure becomes mandatory and this topic is also considered.
\end{abstract}

\section{Introduction}

The influence of strong magnetic fields on the QCD phase diagram is a topic of intense investigations both with lattice and effective model calculations. The whole $T-\mu$ plane was studied [1] with the Nambu-Jona-Lasinio model [2] and it was shown that the first order segment of the transition line becomes longer as the field strength increases so that a larger coexistence region for hadronic and quark matter should be expected for strong magnetic fields. The location of the critical end point is also affected by the presence of magnetic fields which invariably increases the temperature value at which the first order line terminates. At low temperatures, the critical chemical potential displays an oscillation around the value where the magnetic field vanishes for magnetic fields within the $10^{17}-$ $10^{20} \mathrm{G}$ range. These findings have non trivial consequences for the physics of magnetars.

Neutron stars generally manifest themselves as pulsars, which are powered by their rotation energy or as accreting X-ray binaries, which are powered by the gravitational energy. Some compact objects, known as magnetars, do not fit into any of these categories. They are normally isolated neutron stars whose main power source is in the magnetic field and two classes have been discovered: the soft gamma-repeaters that are x-ray transient sources and the anomalous $\mathrm{x}$-ray pulsars, a class of persistent $\mathrm{x}$-ray sources with no sign of a binary companion. Hence, magnetars are extremely magnetized neutron stars, with magnetic fields reaching $B=10^{15} G$ at the surface and central magnetic fields that could reach even higher values [3].

In the present work we check the possibility that magnetars can be quark stars, based on the Bodmer-Witten conjecture [4] that gives a prescription for the calculation of stability windows. These windows are identified with the model parameters that are consistent with the fact that two-flavor quark matter must be unstable (i.e., at zero temperature its energy per baryon has to be larger than 930

a e-mail: debora.p.m@ufsc.br

This is an Open Access article distributed under the terms of the Creative Commons Attribution License 2.0, which permits unrestricted use, distribution, and reproduction in any medium, provided the original work is properly cited. 
$\mathrm{MeV}$, the iron binding energy) and three-flavor quark matter, also known as strange matter (SM) must be stable (i.e., its free energy per baryon must be lower than $930 \mathrm{MeV}$ ), for different models that are generally applied to describe quark stars: the Quark Mass Density Dependent model (QMDD) and the MIT bag model [5]. If finite size effects are taken into account, the binding energy value moves to 934 $\mathrm{MeV}$. In the present work, these surface effects are neglected because they were shown in [5] to change very little the overall results. The QMDD model [6] is based on a phenomenological approach where the dynamical masses of the three lightest quarks scale inversely with the baryon number density $n_{B}$ :

$$
m_{u, \bar{u}}^{*}=m_{d, \bar{d}}^{*}=\frac{C}{3 n_{B}}, \quad m_{s, \bar{s}}^{*}=m_{s, \bar{s}}+\frac{C}{3 n_{B}},
$$

where $C$ is the constant energy density in the zero quark density limit. With a simple prescription, the model mimics asymptotic freedom and confinement. When the MIT model is considered, the quark masses are fixed.

\section{Stability windows and magnetic field effects}

We start from the simpler case, where no magnetic fields are considered and obtain the stability windows for both models. In the left panel of figure 1 left display the results for the QMDD model in two situations: the one normally used in the literature, where the binding energy is used also at finite temperature, and the correct choice, where the free energy is the thermodynamic quantity to be analysed. The shaded area corresponds to the zero temperature case. Note that considering the free energy per baryon as the criterion for stability, the windows move right with the increase of the temperature, Then, we choose the strange quark mass as $m_{s}=100 \mathrm{MeV}$ and show the ranges obtained for various temperatures in the right panel. Similar analysis can be performed for the MIT bag model [7].
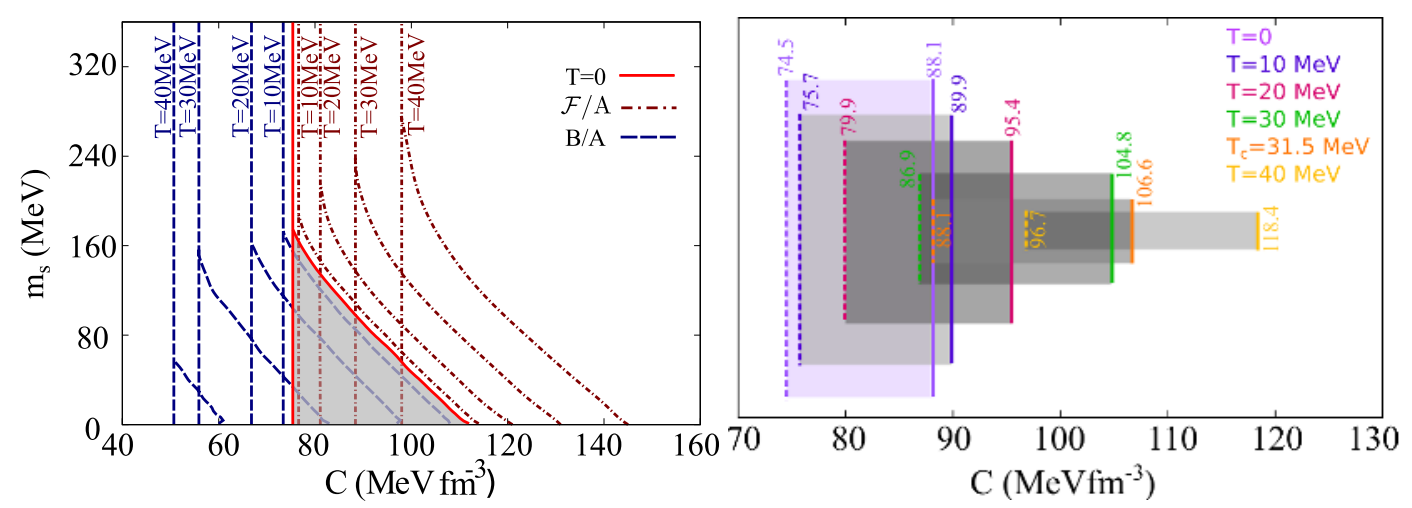

Figure 1. Stability windows of SM obtained with the QMDD model (left) through the analysis of the free energy per baryon and the binding energy per baryon and (right) for parametrization $\mathrm{C}$ (see text) shown for different temperatures.

We then take advantage of the simplicity of the MIT bag model and include magnetic field effects in the calculation of the stability window. The detailed expressions are given in [7] and doing a similar calculation for the QMDD model is straightforward. The equations of state (EOS) are then calculated with the following parameters that respect SM stability: for the MIT (set A), $m_{s}=150 \mathrm{MeV}$ and the 
Table 1. Output results given by the numerical solution of Tolman Oppenheimer Volkoff (TOV) equations, where $B_{c}$ and $\epsilon_{c}$ refer to the central magnetic field and energy density respectively.

\begin{tabular}{cccccccc} 
Set & Model & $S / A$ & $Y_{l}$ & $B_{c}\left(10^{18} \mathrm{G}\right)$ & $M_{\max }\left(\mathrm{M}_{\odot}\right)$ & $R(\mathrm{~km})$ & $\epsilon_{c}\left(\mathrm{fm}^{-4}\right)$ \\
\hline A & MIT & 0 & - & 0 & 1.62 & 9.01 & 8.25 \\
A & MIT & 1 & 0.4 & 0 & 1.64 & 9.10 & 7.96 \\
A & MIT & 2 & 0.4 & 0 & 1.65 & 9.15 & 7.85 \\
A & MIT & 0 & - & 6.64 & 2.02 & 9.04 & 8.31 \\
A & MIT & 1 & 0.4 & 4.71 & 1.95 & 9.05 & 8.82 \\
A & MIT & 2 & 0.4 & 4.44 & 1.93 & 9.08 & 8.69 \\
\hline B & QMDD & 0 & - & 0 & 2.28 & 12.05 & 4.56 \\
B & QMDD & 1 & 0.4 & 0 & 2.31 & 12.16 & 4.39 \\
B & QMDD & 2 & 0.4 & 0 & 2.33 & 12.19 & 4.46 \\
C & QMDD & 0 & - & 0 & 2.26 & 11.76 & 4.65 \\
C & QMDD & 1 & 0.4 & 0 & 2.28 & 11.75 & 4.76 \\
C & QMDD & 2 & 0.4 & 0 & 2.29 & 11.76 & 4.79 \\
\hline
\end{tabular}

bag constant is $155 \mathrm{MeV}^{4}$; for the QMDD (set B), $m_{s}=150 \mathrm{MeV}$ and $C=78 \mathrm{MeV} \mathrm{fm}^{-3}$ and for the QMDD (set C), $m_{s}=100 \mathrm{MeV}$ and $C=85 \mathrm{MeV} \mathrm{fm}^{-3}$. Three snapshots of the star evolution are considered: $S / A=2,1,0, Y_{l}$ is the lepton fraction, which is fixed, except when $S=0$. In this case, no neutrinos are considered and the lepton fraction is not fixed. The results for the MIT and QMDD models are shown in Table 1, from where it is seen that the MIT model can only describe massive stars, as the ones recently observed, if strong magnetic fields are considered. On the other hand, the QMDD model can successfully describe these stars, even if they are not magnetars.

Finally we have chosen the MIT bag model and reobtained its EOS through an anisotropic energy momentum tensor [8]. Due to the magnetic field, the perpendicular component of the matter contribution to the pressure is modified. One can show that the difference of the parallel and perpendicular components of the pressure is proportional to the magnetization that can be calculated by taking the negative of the derivative of the grand potential with respect to the magnetic field $M=-\frac{\partial \Omega}{\partial B}$. The perpendicular component of the matter contribution to the pressure becomes

$$
P_{m_{\perp}}=P_{m_{\|}}-M B
$$

Having established the existence of pressure anisotropy in the matter contribution via Eq. (2), we now turn to the pure-field contributions to the energy-momentum tensor. Due to the presence of the external magnetic field, there are contributions from the electromagnetic field tensor to the pressures and energy density. Due to the breaking of rotational symmetry by the magnetic field, these contributions are again different in the parallel and perpendicular directions. The resulting pressures and energy density read:

$$
\epsilon=\epsilon_{m}+\frac{B^{2}}{8 \pi}, \quad P_{\perp}=P_{m_{\perp}}+\frac{B^{2}}{8 \pi}, \quad P_{\|}=P_{m_{\|}}-\frac{B^{2}}{8 \pi} .
$$

In Fig. 2 we show the differences in both pressures for matter only (left panel), and when the contribution from the pure magnetic field is also included (right panel) for the three snapshots mentioned above labeled i) $S / A=1$, ii) $S / A=2$ and iii) $S / A=0$, except that for $S / A=2$ no neutrinos are considered either. We have taken fixed baryonic chemical potentials, which correspond to intermediate densities $\left(\mu_{B}=1200 \mathrm{MeV}\right)$ and to densities close to the center of the stars $\left(\mu_{B}=1500 \mathrm{MeV}\right)$. In the left panel the discrepancies appear only at very large magnetic fields, but the right panel shows clearly 

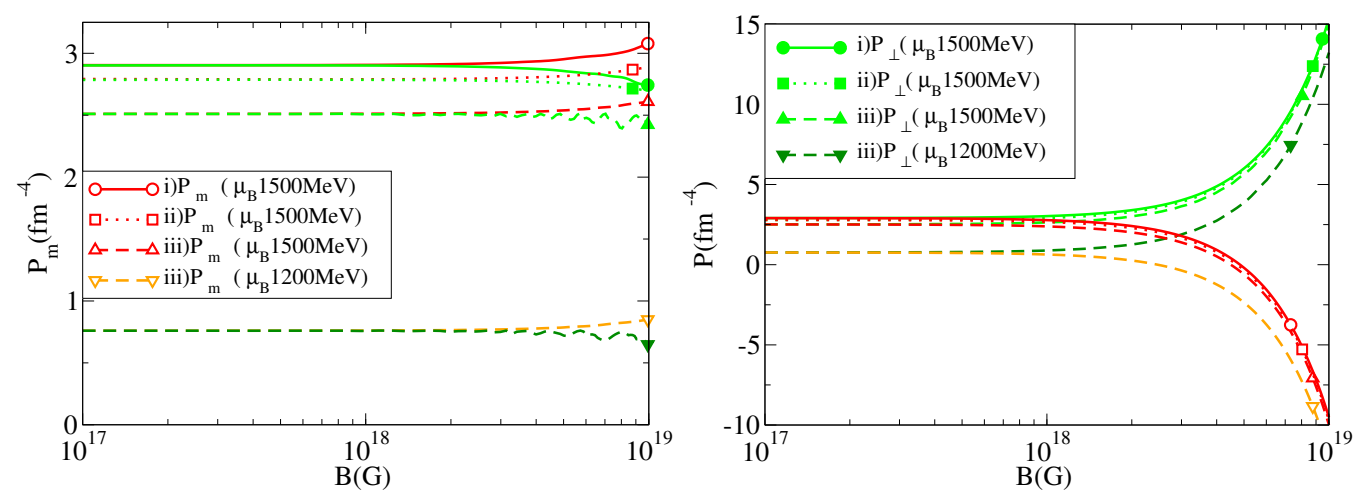

Figure 2. (Parallel (open symbols on red/orange lines) and perpendicular (respective full symbols on green/ dark green lines) pressures of matter versus magnetic field representing different snapshots of the star evolution for (left) without pure magnetic field contribution and (right) with pure magnetic field contribution included.

that results obtained from isotropic equations of state as input to the TOV equations when magnetic fields are taken into account have to be regarded with caution.

\section{Conclusions}

Our results show that if stable quark matter is properly obtained from the stability windows, the QMDD model can account for massive protoquark stars. The simpler MIT bag model can only describe massive stars if subject to strong magnetic fields.

When pressure anisotropy is investigated at different possible stages of the protoquark star evolution, one sees that all results obtained from isotropic equations of state have to be taken as an approximation for the evaluation of qualitative results, since the differences between the perpendicular and parallel pressures are not negligible.

Acknowledgments: This work was partially supported by CNPq, Capes and FAPESC/Brazil.

\section{References}

[1] S.S. Avancini, D.P. Menezes, M.B. Pinto and C. Providência, Phys. Rev. D 85, 091901(R) (2012).

[2] Y. Nambu and G. Jona-Lasinio, Phys. Rev. 122, 345 (1961).

[3] G. Kouveliotou et al., Nature 393, 235 (1998); R. C. Duncan, C. Thompson, ApJ L9,392(1992); ApJ 469, 764 (1996).

[4] N. Itoh, Prog. Theor. Phys., 44, 291 (1970); A.R. Bodmer, Phys. Rev. D 4, 1601 (1971); E. Witten, Phys. Rev. D 30, 272 (1984).

[5] J.R. Torres and D.P. Menezes, Europhys. Lett. 101, 42003 (2013).

[6] S. Chakrabarty, Phys. Rev. D 43, 627 (1991); G. Lugones and O.G. Benvenuto, Phys. Rev. D 52, 1276 (1995); G.X. Peng, H.C. Chiang, B.S. Zou, P.Z. Ning and S.J. Luo, Phys. Rev. C 62, 025801 (2000).

[7] V. Dexheimer, J.R. Torres and D.P. Menezes, Eur. Phys. Jour. C 73:2569 (2013).

[8] M. Strickland, V. Dexheimer and D.P. Menezes, Phys. Rev. D 86, 125032 (2012); V. Dexheimer, D.P. Menezes and M. Strickland, J. Phys G: Nucl. Part. Phys.(2013) in press; arXiv:1210.4526 [nucl-th]. 